\title{
$1949-1976$ 年地极长期运动的显著性问题
}

\author{
李正心 \\ (中国科学院上海天文台)
}

当将地极的长期运动和平纬的变化分别表示成:

和

$$
\left.\begin{array}{rl}
x & =x_{0}+p\left(t-t_{0}\right)+g, \\
y & =y_{0}+q\left(t-t_{0}\right)+h \\
\varphi_{i} & =a_{i}+b_{i}\left(t-t_{0}\right)+f_{i}
\end{array}\right\}
$$

时, 长期极移沿坐标轴方向的线性运动速度 $p 、 q$ 仅仅与平纬序列中线性项的系数 $b_{i}$ 有关, 即 存在关系式 ${ }^{[1]}$ :

$$
M S=B,
$$

其中

$$
M=\left\{\begin{array}{ccc}
\cos \lambda_{1} \sin \lambda_{1} & 1 \\
\cdots & \cdots & \ldots \\
\cos \lambda_{n} \sin \lambda_{n} & 1
\end{array}\right\}, \quad S=\left\{\begin{array}{c}
p \\
q \\
r
\end{array}\right\}, \quad B=\left\{\begin{array}{c}
b_{1} \\
\vdots \\
b_{n}
\end{array}\right\} .
$$

$r$ 一一对所有台站共有的一个系数. 在 (1) 和 (2) 式中, $g 、 h$ 和 $f_{i}$ 都是时刻 $t$ 的非线性 函数.

Федоров ${ }^{[1]}$ 利用上述关系式，根据 ILS 系统 $\mathrm{M} 、 \mathrm{C} 、 \mathrm{G} 、 \mathrm{U}$ 四个平纬序列的 $b_{i}$, 对在 1900-1969 期间是否存在着长期极移的可能性进行了研究，最后得到了“不反对 M、C、G、U 的 $b_{i}$ 是随机性的值的这一假设”的结论. Yatskiv ${ }^{[2]}$ 也提出, 如果仅限于 ILS 系统的五个公 站,是无法分离各 $b_{i}$ 中的极性和非极性成分的.

在这里,我们利用 ILS 和其它众多的独立台站的测纬资料来进一步研究这个问题. 表 1 列出了全球 31 个平纬序列, 其资料来源及处理方法均与文献 [3] 采用的相同. 其中 1-11 台 站是在 1949.0-1976.9 的 28 年间始终有观测的长序列. 其它 12-31 台站开始观测的时刻则 迟于 1949 年, 但它们到 1976.9 年时的观测资料长度都至少在 14 年以上. 它们的 $b_{i}$ 值是这 样得到的: 根据对这些平纬序列谱结构研究的结果 ${ }^{[4]}$, 知道这些平纬序列中还普遍存在着四 种长雕期项(其平均值为 6.4 年、 4.6 年、3.5 年和 2.6 年); 现在按最小二乘法把这四个凬期项 与线性项同时拟合, 就得到了各个平纬序列的系数 $b_{i}$.

根据表 1 所列数据对(3)式所示的这样一个二元线性回归分析问题进行计算和方差分析. 对 1-5 台站的 ILS 系统和 1-11 台站的 1.1 台站系统分别进行计算, 所得 1949.0-1976.9 年: 期间长期极移的线性量和 $\mathrm{F}$ 值 (回归均方与剩余均方之比)为:

本文1981年3月11日收到。 


\begin{tabular}{|c|c|c|}
\hline & ILS 系统 & 11 台站系统 \\
\hline $\begin{array}{l}\text { 线性量的速率 } v \text { : } \\
\text { 线性量的方向 } \theta \text { : } \\
\text { 回归显著性检猃: }\end{array}$ & $\begin{array}{c}0 . .^{\prime} 00512 \pm 0 . .^{\prime \prime} 00468 / \text { 年 } \\
104 .{ }^{\circ} 4 \pm 39 .{ }^{\circ} 7 \mathrm{~W} \\
F=1.49 \\
F_{2 ; 2}^{0.10}=9.00\end{array}$ & $\begin{array}{c}0 . .^{\prime} 00488 \pm 0 . .^{\prime \prime} 00201 / \text { 年 } \\
105 .{ }^{\circ} 2 \pm 17 .{ }^{\circ} 6 \mathrm{~W} \\
F=7.21^{*} \\
F_{2 ; 8}^{0.05}=4.46 \\
F_{2 ; 8}^{0.01}=8.65\end{array}$ \\
\hline
\end{tabular}

表 1 全球 31 个平纬序列 $b_{i}$ 值一览表 （ $b_{i}$ 值单位: $0.00001 /$ 年）

\begin{tabular}{|c|c|c|c|c|c|c|c|c|}
\hline No. & 1 & 2 & 3 & 4 & 5 & 6 & 7 & 8 \\
\hline 台站名称 & $M Z Z$ & $\mathrm{KZ}$ & $\mathrm{CA}$ & GI & UK & POZ & $\mathrm{BL} . \mathrm{Z}$ & MZL \\
\hline 资料长度 $\mathbf{l}_{\mathbf{i}}$ (10 年) & 2.8 & 2.8 & 2.8 & 2.8 & 2.8 & 2.8 & 2.8 & 2.8 \\
\hline 经度 (度) & -141.1 & -66.9 & -8.3 & 77.2 & 123.2 & -34.5 & -20.5 & -141.1 \\
\hline$b_{\mathbf{i}}$ & -424 & -365 & -030 & 039 & 1000 & -348 & -236 & 051 \\
\hline $\mathrm{v}_{\mathbf{i}}$ & 442 & 121 & 21 & 494 & -427 & 192 & 152 & -33 \\
\hline$\Delta_{\mathbf{i}}$ & 681 & 681 & 681 & 681 & 681 & 681 & 681 & 681 \\
\hline No. & 9 & 10 & 11 & 12 & 13 & 14 & 15 & 16 \\
\hline 台站名称 & PUZ & W & $\mathrm{RCP}$ & OS & TO & PA & $M Z P$ & G \\
\hline 资料长度 $1_{\mathrm{i}}(10$ 年) & 2.8 & 2.8 & 2.8 & 2.5 & 2.3 & 2.3 & 2.0 & 1.9 \\
\hline 经度 (度) & -30.2 & 77.1 & 80.4 & 75.7 & -139.5 & -2.3 & -141.1 & -0.3 \\
\hline$b_{i}$ & 023 & 870 & 315 & 366 & 694 & 210 & -310 & 429 \\
\hline$v_{i}$ & -159 & -338 & 229 & 161 & -687 & -178 & 327 & -384 \\
\hline$\Delta_{i}$ & 681 & 681 & 681 & 762 & 829 & 829 & 953 & 1003 \\
\hline No. & 17 & 18 & 19 & 20 & 21 & 22 & 23 & 24 \\
\hline 台站名称 & PrA & $\mathrm{N}$ & $\mathrm{BK}$ & $\mathrm{TJ} Z$ & $\mathrm{ZIA}$ & $\mathrm{BJ} A$ & $\mathrm{D}$ & $\begin{array}{l}\text { Engel } \\
\text { hardt }\end{array}$ \\
\hline 资料长度 $1_{i}$ (10 年) & 1.9 & 1.9 & 1.8 & 1.8 & 1.7 & 1.5 & 1.5 & 1.5 \\
\hline 经度 (度) & -13.1 & -7.0 & -127.0 & -117.0 & -121.4 & -116.3 & -13.8 & -48.8 \\
\hline$b_{i}$ & -573 & 302 & 010 & 018 & 018 & -547 & 439 & 318 \\
\hline$v_{i}$ & 533 & -302 & -79 & -145 & -122 & 416 & -483 & -528 \\
\hline$\Delta_{i}$ & 1003 & 1003 & 1059 & $105^{\prime}$ & 1121 & 1271 & 1271 & 1271 \\
\hline No. & 25 & 26 & 27 & 28 & 29 & 30 & 31 & \\
\hline 台站名称 & $\mathrm{H}$ & $1 R Z$ & $\mathrm{~KB}$ & $\mathrm{UA}$ & Warsă w & $\mathrm{BOZ}$ & $A L$ & \\
\hline 资料长度 $\mathrm{l}_{\mathrm{i}}$ (10 年) & 1.5 & 1.5 & 1.5 & 1.5 & 1.5 & 1.5 & 1.4 & \\
\hline 经度 (度) & -10.0 & -104.3 & -66.9 & -4.3 & -21.0 & -17.1 & -3.0 & \\
\hline$b_{i}$ & 619 & -320 & -164 & -284 & -273 & -096 & 058 & \\
\hline$v_{i}$ & -639 & 136 & -080 & 302 & 186 & 032 & -031 & \\
\hline$\Delta_{\mathbf{i}}$ & 1221 & 1271 & 1271 & 1271 & 1271 & 1271 & 1361 & \\
\hline
\end{tabular}


上述计算结果说明, 在研究 1949-1976 年期间的长期极移问题时,如果单纯由 ILS 五个 台站的测纬资料进行, 虽然可以得到长期极移线性项的速率和方向, 但是从统计推断的理论 来看, 由于所得的 $F$ 值远远小于显著性水平为 0.10 的 $F_{2,2}^{0.10}=9.00$ 的要求, 因而不能认为这 个结果是有意义的. 这一点与上述 Федоров 的研究结论 ${ }^{[1]}$ 是一致的. 但是如果 取 $1949-$ 1976 年期间有观测的 11 个平纬序列来研究这个问题时, 所得结果达到了显著性水平为 0.05 时的要求. 这就说明, 由数量较多而又具有一定长度的经典天文纬度序列, 还是有可能对长期 极移的主要分量(线性分量)进行研究的.

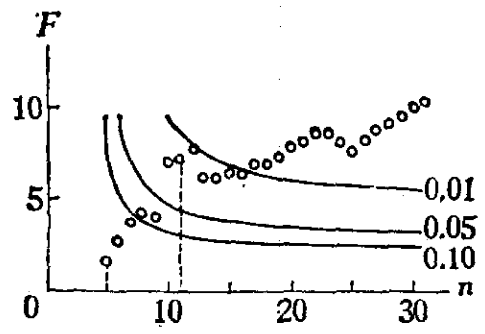

图 1 台站数目 $n$ 增大时 $F$ 值的变化示意图

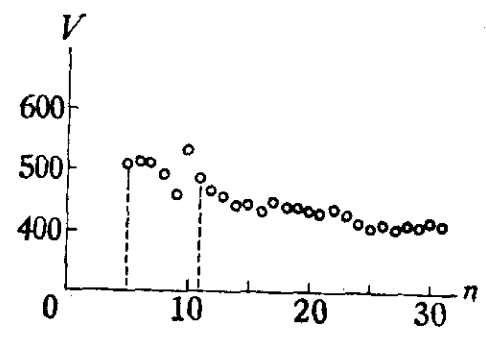

图 2 台站数目 $n$ 增大时 $v$ 值的变化示意图

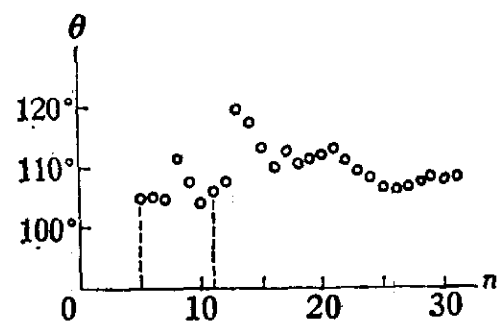

图 3 台站数目 $n$ 增大时 $\theta$ 值的变化示意图

为进一步理解这个问题, 有必要专门考察一下台站的组成, 特别是台站的数目, 以及纬度 序列的长度等因素, 究竟可能对长期极移的研究产生什么样的影响?

首先研究台站的数目对长期极移研究的影响. 为了扩大台站数目可能变动的范围, 现在 把表 1 中 12-31 台站的 $b_{i}$ 值也用进去. 由于这些台站的资料长度不足 28 年而又参差不齐， 所以不能再采用等权的处理方法, 而命各 $b_{\mathrm{i}}$ 值的权 $p_{i}=l_{i}{ }^{2}$. 图 1 、图 2 和图 3 分别表示在 按表 1 中台站编号的次序 (这个次序是按平纬序列的长度排列的, 并没有特别的人为因素), 由 ILS 系统(即 $1-5$ 台) 逐步扩大到 $1-6, \cdots \cdots 1-31$ 台站系统时, $F$ 值、长期极移线性项的速率 $V$ 和方向 $\theta$ 的变化情况. 图 1 说明, 就上述这样一个实际例子而言, 在台站的数目 $n$ 达到了 $8 、 10 、 17$ 以后，回归分析的显著性水平也分别稳定地达到了 $0.10 、 0.05$ 和 0.01 的要求. 这说 明,像其它的回归分析问题一样,采用过小的子样往往可能影响到对问题的分析. 像对本文讨 论的这样一个具体问题而言, 当测纬资料长度达到 30 年这样一个数量级时, 子样数(台站数) 不小于 20 看来是有其必要的.

图 2 和图 3 则说明, 就上述这样一个具体例子而言,台站数目 $n$ 的变化并没有对长期极移 线性项的计算结果表现出严重的影响.

在表 1 各台站数据的最后二行还列出了在 1-31 台站一起进行回归分析计算时所得的残 差 $V_{i}$ 和相应的限差 $\Delta_{i}$ （根据 


$$
\Delta_{i}=2.6-\frac{\mu}{\sqrt{p_{i}}}
$$

计算. 其中的单位权中误差 $\mu$ 根据表 1 列出的 31 个台站的残差 $V_{i}$ 及相应的 $p_{i}$ 按

$$
\boldsymbol{\mu}= \pm \sqrt{\frac{[p v v]}{n-t}}
$$

计算的. 现得 $\mu= \pm 733$ ). 不仅在表 1 中,而且在图 1-3 所示的整个计算过程中, 都没有 发现有 $V_{i}$ 超过相应 $\Delta_{i}$ 的现象. 因此可以认为上述 31 个台站的 $b_{i}$ 都属于同一母体而不宜 再进行取舍. 这也同时说明, 在上述这些台站的范围内, 当台站的总数不是过小时, 台站组成 的不同将不致严重地影响对长期极移的研究.

现在再顺便考察一下资料长度的变化对 $F$ 值的影响. 图 4 是表 1 中 31 个台站统一取

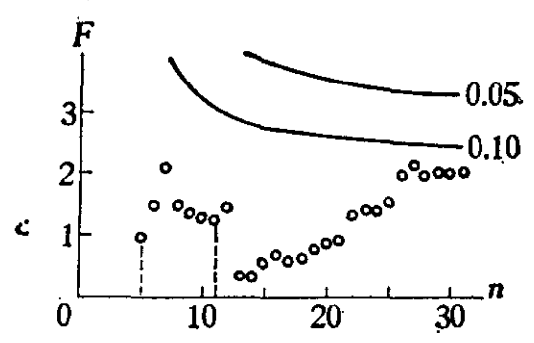

图 4 台站数目 $n$ 增大时 $F$ 值的变化示意图

平纬序列统一取 1963.0-1976.9

1963.0-1976.9 年 14 年的平纬序列时, 由图 1 所示的同样方法情况下所得到的计算结果. 它 表明,在平纬序列的长度一律缩短到 14 年以后, 当台站的数目增大到 $n=31$ 时, 回归分析的 显著性水平仍没有能达到 0.10 的要求. 这再次说明, 在考虑长期极移的研究方案时, 台站的 数量 (子样的大小)和纬度序列的长度 (子样中观测数据的精度)这两者必须联系起来考虑. 像 上述长度为 14 年的天文纬度序列, 当进一步大大增加台站的数目, 才有可能能对相应期间的 长期极移作出有意义的研究.

致谢: 本文在写作过程中,南京大学天文系许邦信副教授曾多次提出宝贵意见,特此致谢.

\section{参考文献}

[1] Федоров Е. П., Астрометрия и астрофизика, 27(1975), 3-6.

[2] Yatskiv, Ya. S., Korsun', A. A., Mironov, N. 'T., IAU Symp., 1979, 82: 29-39.

[3] 极移协作小组, 天文学报, 20 (1979), 1: 1-8.

[4] 李正心,天文地球动力学文集, 1979, 125-132. 